\title{
Selected Abstracts of the TEEPGI 2013 Workshop (1st to 3rd March 2013)
}

\section{A Case of Paraprosthetic Leak diagnosed with the Help of 3D TEE}

Rakendra Singh, Rohit Tandon, S Kumbhkarni, Naved Aslam, Bishav Mohan, PL Gautam, GS Wander

Hero DMC Heart Centre and Dayanand Medical College and Hospital, Ludhiana, Punjab, India

Background: Paravalvular or paraprosthetic leak (PVL) is a complication associated with the surgical/transcatheter implantation of a prosthetic heart valve, more commonly a mechanical than a bioprosthetic valve. Transthoracic echocardiography (TTE) is the most important diagnostic modality for prosthetic valve function/dysfunction. However, TTE often cannot differentiate the PVL from prosthetic regurgitation. Transesophageal echocardiography (TEE) is the modality of choice which is also able to detect small, nonsignificant jets.

Case Report We present a case of 78-year-old male who had undergone mitral valve replacement with E pic St. J ude tissue valve 3 years back and now presented to our hospital with NYHA class III dyspnea since few months prior to admission. It was associated with cough with mucoid expectoration along with orthopnea. TTE revealed moderate PAH with increased gradient across bioprosthetic mitral valve with suspicion of peravalvular leak (Fig. 1). Three-dimensional (3D) volume rendering image analysis after cropping suggested oval perivalvular area of leakage which was confirmed with the help of 3D color rendering (Fig. 2). It showed early separation of perivalvular leak from paravalvular area.

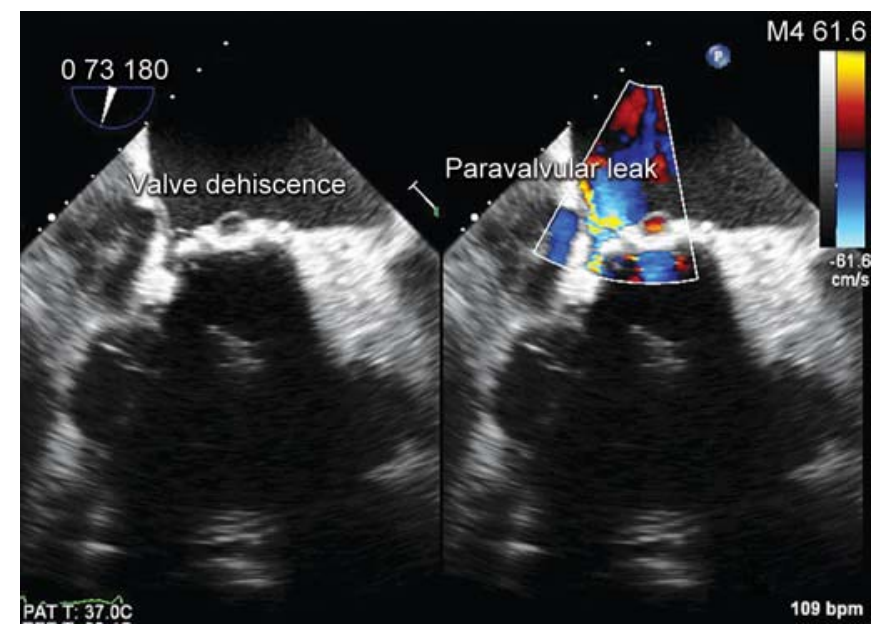

Fig. 1: Transesophageal echo showing paravalvular area of dehiscence along with the central orifice of mitral valve. Second part shows better delineation on color flow rendering

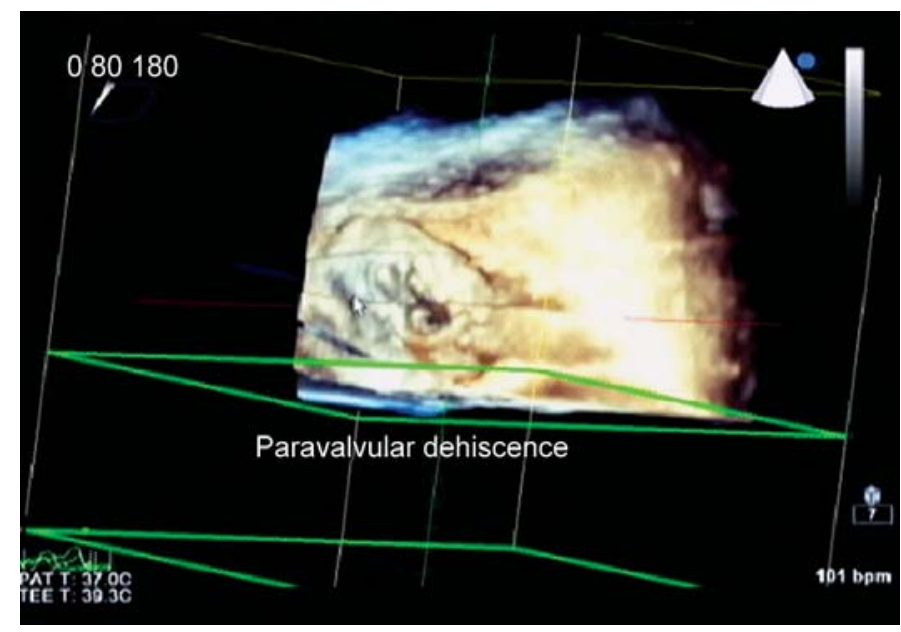

Fig. 2: 3D reconstruction of TEE images delineates paravalvular leak

Discussion: The majority of PVL are crescent, oval or roundish-shaped and their track can be parallel, perpendicular or serpiginous. Incidence of PVL, including small nonsignificant jets, is estimated to be as high as $20 \% .^{1}$ TEE is more useful than TTE in evaluating the shape of paravalvular defect. It may also help in assessing eccentric jets and differentiating between single and multiple jets. Major progress in TEE in the area of PVL imaging has been $3 \mathrm{D}$ reconstruction. ${ }^{2} \mathrm{~A}$ semiquantitative assessment of the size of PVL may be made by measurement of the ratio of total sewing ring circumference to the length of suture dehiscence (<10\%: Mild, 10-20\%: Moderate, $>20 \%$ : Severe, $>40 \%$ : Instability of the prosthetic valve). ${ }^{3}$

Conclusion: The TEE, especially if 3D volume rendering is used along with, provides a systematic method to accurately localize native and prosthetic mitral valve regurgitant lesions. Also, paravalvular leaks may be more easily delineated and quantified using 3D echo. Due to accurate localization of the defects, this modality helps to improve the preoperative assessment and decisions about patients with significant mitral regurgitation.

\section{REFERENCES}

1. Pate GE, AI Zubaidi A. Percutaneous closure of prosthetic paravalvular leaks: Case series and review. Catheter Cardiovasc Interv 2006;68:528-33.

2. Hamilton-Craig $\mathrm{CH}, \mathrm{Boga} \mathrm{T}$. The role of $3 \mathrm{D}$ transesophageal echocardiography during percutaneous closure of paravalvular mitral regurgitation. J A m Coll Cardiol Img 2009;2:771-73.

3. Nietlispach $\mathrm{F}$, J ohnson $\mathrm{M}$. Transcatheter closure of paravalvular defects using a purpose-specific occluder. J A m Coll Cardiol Intv 2010;3:759-65. 


\section{Severe Aortic Stenosis with Reduced Ejection Fraction- Will Left Ventricle Performance improve after Surgery?}

\section{Ravi Raj, Aveek Jayant, GD Puri}

Department of Anesthesia and Intensive Care, Postgraduate Institute of Medical Education and Research, Chandigarh, India

Background: The left ventricular systolic performance is usually maintained till late in patients with aortic stenosis. Echocardiography derived low ejection fraction in patients with severe aortic stenosis can be due to two different reasons: Afterload mismatch due to excessively high wall stress or myocardial dysfunction itself. Patients with afterload mismatch as the predominant mechanism have better outcome after aortic valve replacement than patients with left ventricular dysfunction.

Case Series: We present a series of three patients with severe aortic stenosis and low left ventricular ejection fraction (LVEF). First patient, a 60 years male with sclerodegenerative aortic valve with severe aortic stenosis and LVEF 15 to $20 \%$ underwent aortic valve replacement with cardiopulmonary bypass (CPB) support. Immediate post-CPB transesophageal echo showed marked improvement in LV systolic performance. Patient had excellent recovery in the postoperative period. Second patient, a case of coronary artery disease, severe aortic stenosis and mild to moderate mitral regurgitation underwent coronary artery bypass grafting and aortic valve replacement. Pre-CPB TEE confirmed preoperative diagnosis of severe aortic stenosis with peak aortic foreward velocity more than $4 \mathrm{~m} / \mathrm{sec}$ (Fig. 1). Postoperatively, patient remained in low cardiac output state and expired on postoperative day 2 . Third patient, a 48 years male with severe aortic stenosis, moderate aortic regurgitation and LVEF 10 to $15 \%$ underwent aortic valve replacement with CPB support. Patient was weaned off CPB with high inotrope and intra-aortic balloon pump support. Postoperatively, he had some improvement in LV function but had prolonged stay in intensive care unit.

Conclusion: Identification of predominant mechanism for low LVEF in patients with severe aortic stenosis can affect surgical decision. The role of dobutamine or vasodilators to distinguish patients with true severe valvular stenosis from mild to moderate stenosis and left ventricle dysfunction will be discussed.

\section{Mitral Regurgitation with Left Atrial Myxoma: Not Always Benign}

\section{Ravi Raj, Bhupesh Kumar}

Department of Anesthesia and Intensive Care, Postgraduate Institute of Medical Education and Research, Chandigarh, India

Background: Left atrial (LA) myxoma usually presents with features of mitral stenosis. Incidence of mitral regurgitation (MR) is rare and is generally thought to be due to the mechanical hindrance in leaflet coaptation by the tumor. Rarely myxoma can cause mechanical traumatic rupture of the chordae. However, the echocardiographic assessment of this and severity of MR may be difficult due to shadowing effect of the tumor. Rarely myxoma may get embolized into the coronary artery causing coronary artery obstruction and its sequel.

Case Report: A 62 years male presented to us with progressive dyspnea on exertion and pedal edema. A preoperative transthoracic echocardiography (TTE) showed a LA mass measuring $25 \times 25 \mathrm{~mm}$ with features suggestive of LA myxoma, mild MR and moderate left ventricular systolic dysfunction. A coronary artery angiogram showed diseased left anterior descending artery with distal cutoff. The right coronary artery although was normal showed very sluggish flow. Inside the operating room, TTE examination before induction of anesthesia

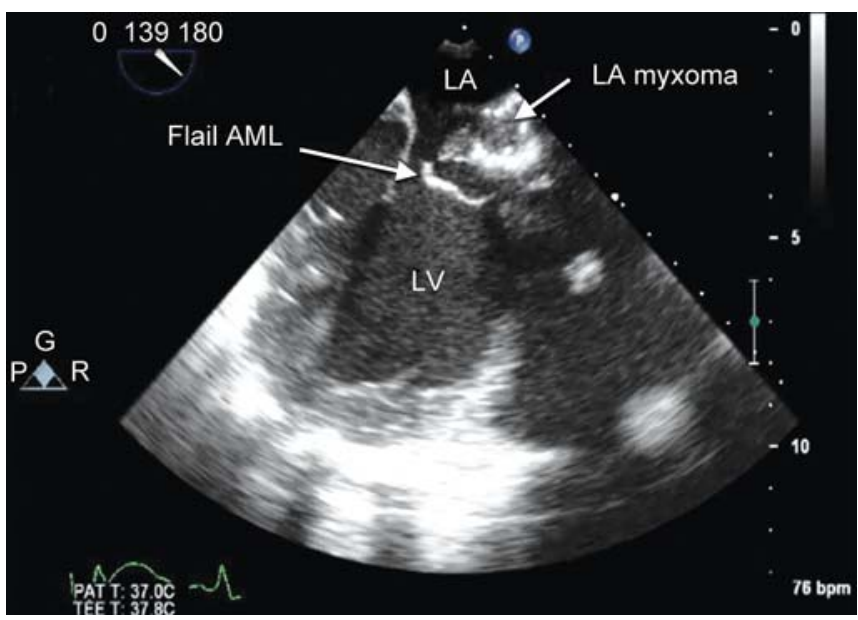

Fig. 1: LA myxoma with flail anterior mitral leaflet due to ruptured chordae 
in apical four-chamber view showed smoke in right atrium and right ventricle with poor right ventricle contractility. There were no features of prolapse or flail mitral valve on two dimensions (2D); however, color Doppler showed severe MR with wall hugging, posteriorly directed eccentric jet. Subsequent close look in modified parasternal long-axis view showed a flail anterior mitral segment which was subsequently confirmed on transesophageal echocardiogram in midesophageal aortic long axis due to ruptured chordae (Fig. 1). Patient required mitral valve replacement in addition to removal of LA myxoma. The intraoperative surgical findings confirmed the echo findings. Nothing was done for coronary as it was thought due to embolization of tumor in distal branch vessels.

Conclusion: We suggest a careful 2D echocardiographic examination in multiple views with application of color Doppler to look for cause of MR and assessment of its severity in patients of LA myxoma as the tumor itself may cause hindrance in examination.

\section{Migrating Intrathoracic Foreign Body: Intraoperative Localization and Monitoring using Transesophageal Echocardiography}

\section{Ashok Kumar Badamali, Bhupesh Kumar, Ishwar Bhukal, Shiva Prasad}

Department of Anesthesia and Intensive Care, Advanced Cardiac Center, Postgraduate Institute of Medical Education and Research Chandigarh, India

Case Report: A 38-year-old male was referred to our cardiac surgical emergency with chief complain of right-sided chest pain and difficulty in breathing for 4 days following a blast injury at industry 1 week back. He was conscious and oriented with stable hemodynamics. Auscultation of chest revealed decreased air entry on right side of chest. Chest radiography showed opacity in right lower half of chest with an intercostal chest tube drain in situ. A contrast-enhanced computerized tomography (CT) performed at the referring hospital showed a $1.6 \times 1.4 \mathrm{~cm}$ metallic foreign body on right-sided paravertebral area in posterior mediastinum, superior to the entry of inferior vena cava into right atrium, with associated hemothorax. CT angiography showed metallic foreign body in posterior mediastinum on right side posterior to left atrium, abutting right inferior pulmonary vein and posterior wall of left atrium. There was no evidence of any active contrast leak or extravasations in relation to right inferior pulmonary vein. All cardiac chambers, main pulmonary artery and its branches appeared normal.

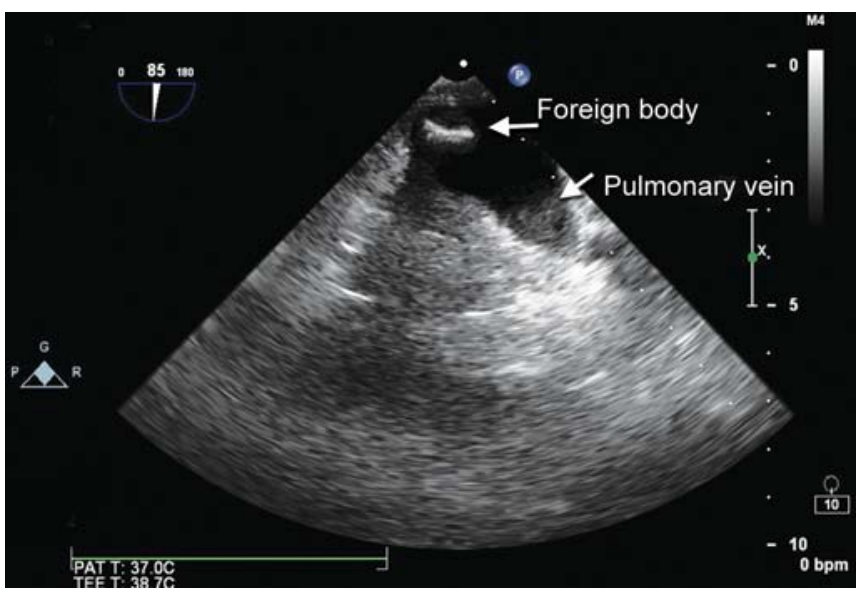

Fig. 1: Foreign body inside left atrium in modified midesophageal two-chamber view

He was taken up for emergency surgical exploration via extended right posterolateral thoracotomy approach in left semilateral position. Following thoracotomy a liter of blood with clots were drained out. The hilum of right lung was exposed and inferior pulmonary ligament was divided. Pericardium was also opened but the surgeon's efforts to locate foreign body based on preoperative chest X-ray and CT report remained futile for hours. Finally, an adult TEE probe was passed through esophagus with the patient remaining in same position. TEE in modified midesophageal-2 chamber view showed the foreign body in the left atrium near the posterior wall (Fig. 1). The surgeon was informed of foreign body location and he could feel it near right inferior pulmonary vein. Decision to use CPB was taken. Right femoral artery, right femoral vein and right atrium were cannulated after achieving full heparinization. By this time, TEE showed that the foreign body has migrated to the left ventricle. It was retrieved from left ventricle through interatrial septum. Patient was weaned from CPB using minimal inotropes. He was extubated after 36 hours of surgery and shifted to ward on day 3. One week later, he required local wound debridement under general anesthesia, which was uneventful and he was discharged from hospital after 15 days. 


\title{
Role of Intraoperative Transesophageal Echocardiography in a Case of Infective Endocarditis
}

\author{
${ }^{1}$ Deepak Borde, ${ }^{1}$ Uday Gandhe, ${ }^{2}$ Kaushal Pandey \\ ${ }^{1}$ Department of Cardiac Anesthesia, PD Hinduja National Hospital and Research Centre, Mahim, Mumbai, Maharashtra, India \\ ${ }^{2}$ Department of Cardiac Surgery, PD Hinduja National Hospital and Research Centre, Mahim, Mumbai, Maharashtra, India
}

\begin{abstract}
Case Report A 66-year-old male without any significant medical history was admitted to authors institute with complaints of lowgrade fever on and off, loss of appetite and weight loss of $5 \mathrm{~kg}$ since 4 months. A cardiology consultation was advised in view of systolic murmur in mitral area.

The $2 \mathrm{D}$ echocardiography revealed vegetation on anterior leaflet of mitral valve $9 \times 6 \mathrm{~mm}$ associated with eccentric jet and severe mitral regurgitation. There was a suspicious lesion on left coronary cusp of aortic valve with moderate aortic regurgitation. Preoperative blood culture was negative for any microorganisms. Other blood investigations were within normal limits. On next day, patient developed severe intra-abdominal pain. The computed tomographic (CT) scan revealed splenic infarct. This was presumed to be embolic phenomenon. The patient was posted for mitral valve replacement with possible aortic valve replacement.
\end{abstract}

Intraoperatively after inducing general anesthesia, a precardiopulmonary bypass transesophageal echocardiography was done. This examination confirmed vegetation on anterior mitral leaflet with perforation in A2 segment of anterior mitral leaflet. A mobile vegetation measuring $8 \times 8 \mathrm{~mm}$ on left coronary cusp was also confirmed (Fig. 1). There was no additional vegetation on any other structure. The decision was made to proceed with double valve replacement with bioprosthesis. The echocardiography findings were confirmed by the surgeon. The patient was weaned off cardiopulmonary bypass without difficulty. Postcardiopulmonary bypass revealed nonobstructed prosthesis with no intra- or paravalvular leak. The patient was shifted out of intensive care unit on day 3 and was discharged on day 9 of operation after uneventful recovery.

Present case report confirms role of perioperative transesophageal echocardiography (recommendation grade I) in confirmation of diagnosis (vegetation on anterior mitral leaflet), recognizing additional pathology (perforation of A2 segment of anterior mitral leaflet), confirming uncertain diagnosis (vegetation on aortic valve) and ascertaining normal functioning of prosthesis after discontinuing patient from cardiopulmonary bypass in a case of infective endocarditis.

\section{Role of Doppler Echocardiography in Patients with Tricuspid Valve Lesions with Aorto-right Atrial Fistula: A Diagnostic Dilemma}

\author{
Ajay Kumar Jha, Vishwas Malik, Usha Kiran \\ Department of Cardiothoracic and Vascular Anesthesia, All India Institute of Medical Sciences, New Delhi, India
}

We describe the case of a 40-year-old male with 8 weeks history of progressive dyspnea, palpitation and nonproductive cough. Transthoracic echocardiography showed rupture of the sinus of valsalva into the right ventricle and tricuspid valve stenosis. We did two-dimensional (2D) and three-dimensional (3D) transesophageal echocardiography in the operating room and found the opening of the ruptured right sinus of valsalva into the right atrium just above the septal leaflet of the tricuspid valve with restricted septal leaflet mobility. We also measured the peak and mean transvalvular gradient across the tricuspid valve which was $40 \mathrm{~mm} \mathrm{Hg}$ and $18 \mathrm{~mm} \mathrm{Hg}$ respectively (Figs 1 and 2). There was no evidence of tricuspid regurgitation. After transaortic closure of the neck of aorto-right atrial fistula, we noticed disappearance of transvalvular gradient across the tricuspid valve with an eccentric jet of tricuspid regurgitation (Figs 3 and 4). Interatrial defects, interventricular defects, aorto-atrial or ventricular fistula and valvular regurgitation produce higher flow across the valve and may produce transvalvular gradient simulating stenosis. But, increase in the gradient invariably remains nonsignificant in interatrial defects, interventricular defects and valvular regurgitation in absence of stenotic valve. 


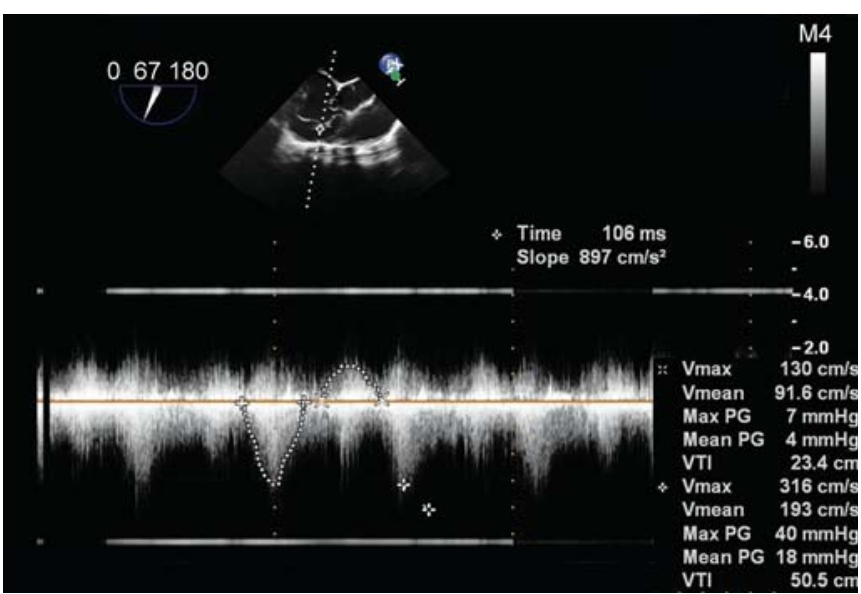

Fig. 1: Continuous wave Doppler across the tricuspid valve in midesophageal RV inflow-outflow view showing peak gradient of $40 \mathrm{~mm} \mathrm{Hg}$ mimicking tricuspid stenosis with trivial or no regurgitation. Opening of the rupture sinus of valsalva is also seen in the right atrium just above the septal leaflet of the tricuspid valve

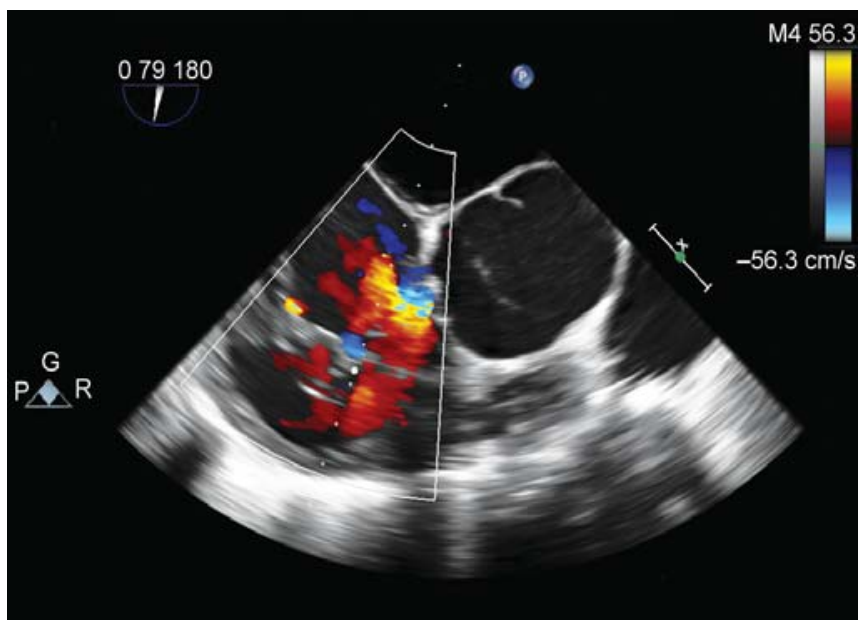

Fig. 3: Eccentric jet of tricuspid regurgitation in midesophageal five-chamber view after the closure of the fistula

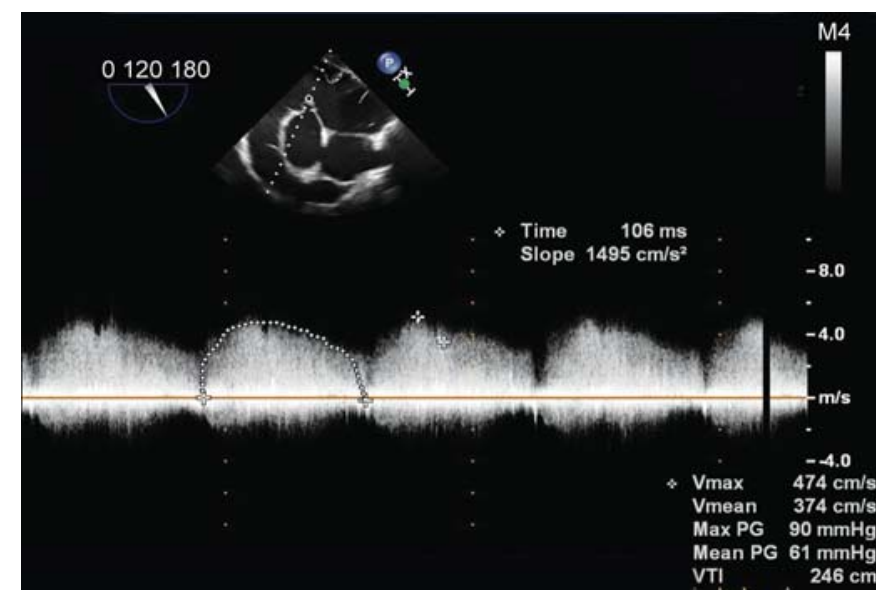

Fig. 2: Continuous wave Doppler across the sites of rupture of the right sinus of valsalva in deep transgastric RV inflow-outflow view

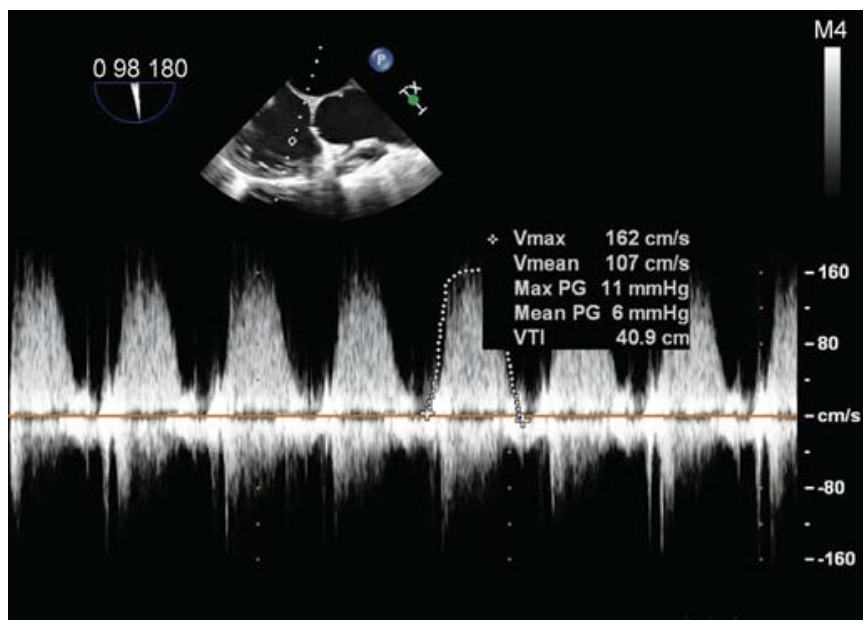

Fig. 4: Continuous wave Doppler across the tricuspid valve shows mild to moderate tricuspid regurgitation with no stenosis (gradient: $1 \mathrm{~mm} \mathrm{Hg}$ ) in midesophageal five-chamber view after the closure of the fistula

Conclusion: 2D and 3D transesophageal echocardiography help in better delineation of the sites of rupture of sinus of valsalva. Determination of transvalvular gradient according to Bernoulli equation $\left[\Delta \mathrm{P}=4\left(\mathrm{v}_{2}^{2}-\mathrm{v}_{1}^{2}\right)\right]$ becomes erroneous in presence of higher flows especially in the aorto-atrial or ventricular fistula because of disproportionate increase in the distal velocity compared to the proximal velocity and thus mimic as tricuspid stenosis. Higher pressure in the proximal chambers of the heart due to excessive blood flow as in aorto-atrial or ventricular fistula masks the presence of valvular regurgitation. 